Nouvelles perspectives en sciences sociales

\title{
Analyse du rôle de l'intention dans les échanges dyadiques
}

\section{Paul Jalbert}

Volume 2, numéro 1, septembre 2006

URI : https://id.erudit.org/iderudit/602456ar

DOI : https://doi.org/10.7202/602456ar

Aller au sommaire du numéro

Éditeur(s)

Prise de parole

ISSN

1712-8307 (imprimé)

1918-7475 (numérique)

Découvrir la revue

Citer cet article

Jalbert, P. (2006). Analyse du rôle de l'intention dans les échanges dyadiques.

Nouvelles perspectives en sciences sociales, 2(1), 101-141.

https://doi.org/10.7202/602456ar d'utilisation que vous pouvez consulter en ligne.

https://apropos.erudit.org/fr/usagers/politique-dutilisation/ 


\title{
Analyse du rôle de l'intention dans les échanges dyadiques
}

\author{
Paul Jalbert \\ Université Laurentienne \\ Sudbury, Ontario, Canada
}

À la base de toute société, on trouve la communication. Elle peut prendre plusieurs formes, mais elle est nécessairement présente. L'omniprésence de la communication est évidente à moins de se trouver dans un contexte d'isolement à perpétuité. Mais un tel scénario est peu probable chez l'humain. Dès la naissance, le bébé communique son incapacité à se défendre ou à se protéger. Ses faiblesses sont immédiatement communiquées aux parents, même en l'absence d'un langage standardisé. Et voilà le début de la vie en société - au fond, la famille.

En plus de constituer un élément constitutif de la vie en société, la communication a favorisé le développement de la société telle qu'on la trouve aujourd'hui. L'un des éléments de la société, telle qu'on la connait, est la standardisation de la communication. On utilise une série de sons, soit seul, soit en groupe, pour transmettre des messages spécifiques.

Cette spécificité se reflète dans la forme que prend la société. Les lois, les politiques et la religion organisée sont tous des exemples du caractère unique de la société et, par conséquent, du langage. La 
complexité et les nuances de la société correspondent à la complexité et aux nuances du langage. Les deux ensembles sont inséparablement liés. L'organisation et la rationalité font partie du caractère fondamental de la société et elles sont la conséquence d'un langage établi. Elles témoignent de ce que les humains sont des êtres relationnels, communiquant à tous moments.

La complexité du langage, ses subtilités en rendent l'étude difficile. La tonalité des mots ainsi que l'expression influencent le message qu'on transmet. Les chercheurs parviennent néanmoins à soulever des hypothèses et à les tester de façon empirique. Le but de ces recherches est d'ériger des théories qui rendent compte de la communication et, plus généralement, du social.

Cependant, on se trouve parfois devant des théories qui surrationalisent le social. Une hypothèse de base veut que l'humain soit rationnel. Cette hypothèse est davantage le reflet de la science utilisée que du phénomène. En d'autres mots, le social n'est peut-être pas aussi rationnel que le veut la science qui approche son objet dans cet esprit. La rationalité de l'acteur social est l'une des qualités qu'on prête au comportement de l'acteur social. Mais ce n'est pas la composante unique dans la communication. Dans le même sens, quand les sciences l'incorporent dans leurs théories, elles se coupent souvent des autres aspects de la socialité, ce qui fait en sorte qu'elles perdent de vue l'essence ou la vérité du social, c'est-à-dire sa complexité.

Une approche phénoménologique dans laquelle s'exprime cette vision rationalisante de l'action humaine n'est pas sans mérite. Mais sa prétention à l'universalité est certainement abusive. On ne peut soutenir bien longtemps que toute personne communique toujours de façon rationnelle et que cette rationalité repose sur le caractère essentiel de l'intention. La complexité du langage n'est pas réductible à cette seule composante. L'acteur social est sans doute rationnel, mais il n'est pas seulement rationnel. Il est beaucoup plus que cela. Il a des motivations intrinsèques aussi bien qu'il subit des influences extrinsèques; il a même des besoins physiologiques : se nourrir ou dormir, par exemple. Certaines forces à l'extérieur cherchent à standardiser ses comportements. Les médias aussi bien que les amis influencent sa manière d'agir. L'influence des médias est bien documentée, celle notamment qui a trait à la publicité. On ne peut pas passer une journée sans rencontrer une annonce publicitaire sous une forme ou un autre. Le but de ces annonces est clair et précis : influencer, convaincre. Durant les 
campagnes électorales, des millions de dollars peuvent être dépensés en annonces publicitaires pour inciter le public à voter d'une façon ou d'un autre. À tout moment, la répétition des annonces publicitaires tente d'orienter le choix des électeurs. L'acteur ne peut y échapper. Les personnes dont il est proche agissent, elles aussi, sur lui. La volonté de plaire aux êtres chers ainsi que l'intimité de la relation font en sorte que les idées et les points de vue deviennent importants. Quand ses proches s'adressent à lui, il les écoute et l'information qui a été captée anime la réflexion. On ne peut éliminer ces influences. Elles sont nécessairement présentes dans notre société.

Le travail qui suit se compose de cinq parties : le contexte théorique, la problématique, la méthodologie, la description des données et leur interprétation de ces données. Nous débuterons par un survol de la perspective phénoménologique en sociologie. Ensuite, nous signalerons certaines limites au paradigme de la phénoménologie, notamment en ce qui concerne les développements théoriques. Ces difficultés évoquées, nous décrirons une méthodologie où nous expliquerons le déroulement de la recherche en précisant la question de la recherche.

\section{Contexte théorique}

\subsection{L'humain : un être relationnel}

L'humain est un être relationnel. Cela l'oblige à entrer en communication avec son entourage. En tant qu'humain, nous communiquons à tout moment. Même lorsque nous décidons de ne pas communiquer, nous communiquons notre volonté de ne pas communiquer ${ }^{1}$. Nous sommes des êtres relationnels et, par conséquent, nous communiquons continuellement. Les omissions aussi bien que les paroles explicites donnent des indices sur la manière dont nous nous sentons, sur ce que nous pensons et sur ce que sont nos intentions. Selon Laflamme ${ }^{2}$, la communication ne peut être comprise que dans cet esprit. Elle a ainsi pour corollaire l'historicité des participants et leur unicité. Elle est aussi associée au contexte de la relation dans laquelle les participants se

1 Paul Watzlawick, Pragmatics of buman communication: A study of interactional patterns, pathologies, and paradoxes, New York, W.W. Norton, 1967.

2 Simon Laflamme, Communication et émotion. Essai de microsociologie relationnelle, Paris, L'Harmattan, 1995. 


\section{NPSS, VOLUME 2, NUMÉRO 1, 2006}

trouvent. Chaque participant apporte avec lui son vécu unique; il porte avec lui ses expériences, son environnement et sa façon de percevoir son environnement. Cela fait en sorte qu'il est un individu unique et qu'il communique de façon unique. Certes, les agents communicants ont aussi un grand nombre de points en commun. Comme l'expliquent Girard, Laflamme et Roggero : «Que l'humain soit social, historique et communicationnel, que son vécu soit complexe, ce sont là des évidences $»^{3}$. L'individu partage avec d'autres des qualités, une histoire collective ; cela lui permet, entre autres, de communiquer. Ce qu'il a en commun avec les autres membres de sa société est forcément important. Cependant, même avec toutes ces similitudes, on ne peut pas contester l'unicité de l'individu ; et elle vaut pour chacun des interlocuteurs. Celui qui écoute est aussi unique que celui qui parle, avec ses expériences, son environnement et la façon dont il perçoit son environnement. Si on accepte que tous les acteurs sont en fait uniques, et que chaque relation est unique, chaque communication entre les participants s'avère unique.

Mais cette unicité est régulée, entre autres par la rationalité. La rationalité permet à un agent communicant d'adopter la perspective d'un autre. La pensée abstraite - par exemple, se mettre dans les souliers d'un autre - donne à chaque individu l'occasion d'adopter le point de vue de l'autre. Nous pouvons apprendre l'histoire d'une personne, connaitre ses peurs et ses rêves sans avoir besoin de les vivre à sa place. Mais avoir une connaissance complète de l'individu n'est pas possible. On ne peut en venir à tout prendre en considération; il manque toujours quelque chose, peut-être plusieurs choses.

Aborder l'acteur en tant que rationnel, c'est là un paradigme des sciences sociales. La rationalité apparaît alors dans tous les replis de l'action et de l'humain. Selon certains chercheurs, la rationalité a pour corollaire l'intérêt. On rationalise en développant une stratégie pour servir ses intérêts. À ce moment, on devient intentionné. Raison, intention, conscience, intérêt ces quatre concepts constituent l'essentiel de l'appareil théorique de la plupart des théories de l'action. En fait, il est peu de théories au sein desquelles l'intention joue un rôle qui ne

Mélanie Girard, Simon Laflamme et Pascal Roggero. "L'intention est-elle si universelle que le prétendent les théories de l'action ? ", Nouvelles perspectives en sciences sociales. Revue internationale de systémique complexe et d'études relationnelles, vol $1, \mathrm{n}^{\circ}$ 2, 2005, p. 115 . 
recourent pas également aux trois autres concepts, et qui, même, ajoutent à la notion d'intention celle de stratégie ${ }^{4}$. Aux yeux de ces théories, l'acteur ou l'agent communicant oriente un projet dans une direction quelconque pour atteindre le but visé. Toutefois, dans la communication, il y a deux acteurs. S'il y a deux acteurs, chacun doit avoir son intérêt propre. Lorsque les deux acteurs tentent de communiquer leurs intérêts, ils ne peuvent les exprimer unilatéralement, sans considération pour l'interlocuteur. Ils doivent suivre des étapes pour s'assurer que le message sera reçu par le destinataire. La communication apparaît alors comme intentionnelle.

Cette intention est tenue pour acquise. Mais l'est-elle vraiment ? La communication n'est pas une relation unidirectionnelle. Une fois la conversation initiée, la communication ne peut être dominée seulement par l'intention. Les participants, et leur unicité qui fait en sorte que leur relation est aussi unique, sont déjà sujets à de nombreuses influences, même sans qu'un mot soit dit, simplement par le fait de leur unicité respective et par le caractère unique de leur échange.

On peut s'imaginer de nombreuses situations où l'intention de départ se perd au fur et à mesure que l'action se développe. La participation de deux locuteurs uniques nécessite non seulement l'application d'une stratégie planifiée et calculée, mais des changements - qui sont appliqués presque instantanément - pour arriver au but envisagé initialement. Lorsqu'il y a dialogue entre participants, l'interaction exige une flexibilité pour que la communication puisse avoir lieu. L'ouverture de la communication est le début. Cependant le discours entre individus, les réactions et l'interaction exigent qu'on réponde à son partenaire. Feltz explique bien la nécessité de la souplesse des stratégies qu'emploie chaque participant :

Une description est toujours associée à un certain projet plus ou moins bien défini ou explicité. Plus généralement encore, ces propos contribuent à la prise de conscience de l'impossibilité d'une description exhaustive d'une situation donnée. Si la description n'est pas exhaustive, cela signifie que des choix sont posés qui distinguent les informations jugées pertinentes de celles qui ne le sont pas.

Ibid., p. 115-148.

Bernard Feltz, La science et le vivant. Introduction à la philosophie des sciences de la vie, Bruxelles, De Boeck, 2003, p. 86. 
On pourrait également faire une comparaison avec une relation amoureuse. Imaginons deux individus qui sont ouverts à une relation. Les deux sont attirés physiquement l'un par l'autre. Ils partagent les mêmes intérêts, buts, rêves et espoirs. La communication entre partenaires pourrait être qualifiée de bonne. Le temps qu'ils passent ensemble est valorisé par chaque partenaire. Cependant, ils ne se sentent pas satisfaits dans la relation. Le projet d'une relation amoureuse et les motivations pour y parvenir sont manifestes, mais certains éléments font défaut. Les amoureux en discutent entre eux, mais ils ne viennent pas à bout de trouver le problème. Les deux expriment leurs sentiments et écoutent attentivement les inquiétudes de l'autre. Après beaucoup de temps et d'efforts, il y a séparation. Les partenaires s'accordent pour dire que la relation ne peut se poursuivre. Dans ce cas, même deux adultes qui s'entendent bien et qui veulent réussir ne mènent pas à terme leur projet. Il doit alors y avoir d'autres éléments, des facteurs qui ne sont pas pris en considération. Et, en fait, même si on pouvait prendre en considération tous les facteurs, la complexité des interactions ne peut être comprise dans sa globalité.

Lorsque deux individus entrent en relation et partagent leur vie, ou bien, simplement communiquent au quotidien, certains éléments rendent l'échange unique. L'histoire personnelle qu'apporte chaque personne, sa façon de communiquer et même sa façon de répondre agissent sur la relation ou sur les échanges. Rousselle $e^{6}$ suggère que même lorsqu'il y a des désaccords entre les partenaires d'un couple, on peut trouver que chaque personne s'accorde sur le fait qu'il y a un désaccord. La communication est efficace tant que le désaccord persiste.

La dynamique d'une relation la rend unique. Elle ne peut être reproduite, puisque les participants ne peuvent être reproduits. Les participants eux-mêmes sont dynamiques. Ils ont leur propre façon d'écouter, de parler, de communiquer. Chaque participant apporte un vécu. Ces vécus ont, en partie, formé l'adulte. Presque toutes les dimensions psychiques de l'adulte ont été influencées par les expériences antérieures. Puisque la communication a des composantes psychiques, on doit s'attendre à ce que ces expériences influencent aussi la façon dont l'individu communique.

6 Jeannine Rousselle, La communication chezles couples. Une approche relationnelle, mémoire de maitrise, Sudbury, Université Laurentienne, 2003. 


\subsection{Complexité et résolution}

De Rosnay $^{7}$ fait une distinction entre deux approches pour faciliter l'analyse ; soit l'approche analytique et l'approche systémique. "L'approche analytique cherche à ramener un système à ses éléments constitutifs les plus simples; afin de les étudier en détail et de comprendre les types d'interactions qui existent entre eux ${ }^{8}$. Cependant, il suggère que des systèmes de haute complexité nécessitent une approche systémique, le but de cette approche étant de : «...considérer un système dans sa totalité, sa complexité et sa dynamique propre $»^{9}$.

Il suggère d'utiliser une approche systémique pour interpréter des systèmes complexes. Il affirme que cette approche, qui date de peu longtemps, est transdisciplinaire et est en mesure de prendre en considération plusieurs éléments d'une organisation complexe. Il décrit cette approche de la façon suivante : "Il ne faut pas la considérer comme une science, une théorie ou une discipline, mais comme une nouvelle méthodologie, permettant de rassembler et d'organiser les connaissances en vue d'une plus grande efficacité de l'action ${ }^{10}$. Comme on peut le constater, cette approche est attachée à la notion de système. Pour de Rosnay : "un système est un ensemble d'éléments en interaction ${ }^{11}$. C'est dans une telle perspective qu'on doit interpréter la communication.

Il poursuit en affirmant que les théories utilisées pour interpréter notre environnement se trouvent souvent trop simplistes, ce qui les rend à la fois utiles et idéales. Mais cet idéal, comme le suggère cet auteur, n'est que cela, un idéal; difficile, sinon impossible à retrouver dans le monde naturel.

Alors on doit croire que l'humain, avec toutes ses complexités, demande une analyse systémique. Par extension, la communication réclame, elle aussi, une approche systémique. La notion d'émorationalité nous semble pouvoir servir une analyse systémique. Laflamme s'explique ainsi quand il propose la notion d'émorationalité : «...on commence par

Joël de Rosnay, Le macroscope. Vers une version globale, Paris, Seuil, 1975.

$8 \quad$ Ibid., p. 118.

9 Ibid.

$10 \quad$ Ibid., p. 91.

$11 \quad$ Ibid., p. 92. 
rappeler le caractère essentiellement socio-historique du vécu humain ; on rapporte, ensuite, ce vécu à l'émotion et à la raison dans leur inséparabilité, d'où naît de la contraction des concepts le terme émoraison... $\gg^{12}$. Dans cet esprit, on pourrait vérifier, en manipulant des variables, s'il est possible, ou plutôt adéquat d'aborder l'humain dans une logique rationaliste. Mais comme Girard ${ }^{13}$ l'a démontré, on ne peut réduire la communication à une forme simpliste, ce qui fait qu'on ne peut modéliser le phénomène de façon trop réductrice. On doit l'interpréter de façon relativement étendue pour enfin la comprendre réellement.

L'approche systémique ouvre certaines avenues. Elle permet de remplacer la rigidité par la souplesse et l'adaptabilité. Mais de quoi l'analyse systémique est-elle composée? De Rosnay explique que «l'analyse de systèmes consiste à définir les limites du système à modéliser ; à identifier les éléments importants et les types d'interactions entre ces éléments, puis à déterminer les liaisons qui les intègrent en un tout organisé $\gg{ }^{14}$.

Dans ce cas, l'importance de chaque élément, ainsi que sa fonction, est relative aux autres composantes. La communication, comme le suggère la notion d'émorationalité, ne peut être interprétée comme un mouvement unilatéral, strictement rationnel et constant ; elle doit être interprétée comme le fait d'une dynamique que produit un ensemble quelconque d'agents communicants.

Cette forme d'analyse laisse aussi croire que le discours ne peut être réduit à l'intentionnalité et à la rationalité. On ne peut se contenter de manipuler une variable pour ensuite en tirer des conclusions; il importe plutôt de faire l'examen de l'ensemble des informations pour tenter de comprendre son fonctionnement. Mais la question se pose de savoir si, dans le cas de la communication, nous pouvons manipuler l'intentionnalité pour manipuler des échanges entre des individus afin de tester l'hypothèse, et non le fait, de l'universalité de l'intention?

Simon Laflamme, Communication et émotion..., op. cit., p. 32.

13 Mélanie Girard, Relations bumaines et production d'information. L'échange comme objet d'étude d'une approche relationnelle, mémoire de maitrise, Sudbury, Université Laurentienne, 2004.

14 Joël de Rosnay, Le macroscope..., op. cit., p. 122. 


\subsection{Théories de l'action humaine}

La phénoménologie, l'utilitarisme ou les théories de l'action humaine jouent un rôle dominant dans les sciences sociales - ils reposent sur des principes communs comme l'affirme Berthelot:

Il y a donc bien, à l'aval des grands principes recteurs et en amont des constructions conceptuelles, un niveau spécifique de la construction de la connaissance. Il est le lieu de ce que nous proposons d'appeler les schèmes d'intelligibilité. De par leur position ceux-ci sont non seulement transdisciplinaires, mais transrégionaux : ils ne sont pas le propre de la connaissance du social et se manifestent également dans les autres domaines ${ }^{15}$.

L'ensemble de ces approches constitue l'un des paradigmes les plus anciens. On en trouve les débuts dans les écrits de Hegel puis dans des textes de Heidegger, de Sartre et de Schultz. À sa base, la phénoménologie suggère qu'à l'arrière-plan de la communication, il y a l'intention.

Dès qu'on commence à examiner les mouvements utilitaristes et phénoménologiques, on se rend compte très rapidement qu'il ne s'agit pas strictement de théories de l'action humaine. Ce sont, en fait, des écoles de pensée. Dans les sciences sociales, ils sont à la source d'une infinité de recherches. En psychologie, en sociologie et, surtout, en économie, cette théorie est tenue pour acquise, ses postulats pour des vérifications : elle ne procède à aucune opérationnalisation pour vérifier si ce qu'elle tient pour des évidences observables correspond à la réalité. Pis encore, cette théorie sert même de point de départ à une multitude de recherches. Cependant, lorsqu'on l'examine de façon critique, on remarque des difficultés.

Le principe de base de cette théorie est le caractère rationnel de l'action humaine, selon lequel chaque décision que prend l'acteur, chaque choix qu'il pose et, plus généralement, tout ce qu'il fait doit, nécessairement, partir d'un raisonnement intéressé. Cette attitude est très clairement expliquée et bien documentée dans l'ouvrage de Girard.

... [S]i les gens sont si heureux dans la libre concurrence (et parallèlement, malheureux en présence du mercantilisme), c'est qu'il existe une espèce de main invisible, une espèce de poursuite 
inconsciente du bonheur de l'ensemble par les particuliers ; ainsi guidé - de façon inconsciente - par ce qui prend la forme d'un intérêt personnel, l'individu s'adonnera à un travail, à une tâche qui contribuera à accroître le revenu annuel de la société. Dans une quête, donc, de gain personnel, l'individu remplirait des buts inconscients, non anticipés ou, plutôt, non intentionnés qui serviraient à assurer le bienêtre de tous. ${ }^{16}$

Cette forme d'égoïsme est manifeste chez Smith et Weber. Alors si l'égoïsme et la poursuite du bonheur sont omniprésents, on doit s'attendre à ce qu'un individu trouve des méthodes pour atteindre son objectif. Ces méthodes ou stratégies sont essentielles à la réalisation de buts, même inconscients! Si on a des objectifs, il est entendu qu'on tente de les atteindre. La volonté de les atteindre peut se définir comme l'intention de l'acteur social. Nous reviendrons un peu plus loin sur ce fait.

Le développement de stratégies suppose l'intention. L'observation de stratégies, par conséquent, incite à conclure que, en premier lieu, l'acteur social a des buts et, en deuxième lieu, il est intentionné. Pour être intentionné - pour viser un objectif - l'acteur social doit d'abord être conscient de lui-même ainsi que de son entourage. Il doit pouvoir se situer dans le temps et dans l'espace. Ce n'est que par ce moyen qu'il peut clairement se fixer un but. Cependant, pour créer des stratégies, l'acteur doit être plus que seulement conscient de lui-même et de son environnement ; il doit être rationnel. Il doit être capable de s'orienter lui-même, d'orienter ses actions en considérant les outils qui lui sont accessibles.

Alors, pour que l'intention puisse se former, le participant doit être nécessairement doué de conscience. De la conscience individuelle découle la raison. Ainsi, pour que l'intention se manifeste dans le discours, elle doit avoir la formulation d'une stratégie. Dans le cas de la communication, ces stratégies sont utilisées pour améliorer l'efficacité de la communication, c'est-à-dire maximiser les gains et minimiser les pertes. Alors, en plus d'être rationnel, l'acteur veut agir à son profit.

En philosophie, on trouve des manifestations de ce point de vue utilitariste. On remarque les premiers indices de l'axiome utilitariste depuis déjà plusieurs siècles. Les écrits de Hobbes et de Hume en sont

16 Mélanie Girard, Relations bumaines et production d'information..., op. cit., p. 8. 
déjà des illustrations. On se rend compte, dès le début de la lecture de leurs ouvres, qu'une logique utilitariste est active.

Hobbes ${ }^{17}$ suggère que notre raison ne sert qu'à réaliser nos désirs. En d'autres mots, la raison est l'outil qu'on utilise pour trouver satisfaction. Les désirs peuvent sans doute conduire à agir. L'individu doit agir pour satisfaire ses besoins. De la même façon, il doit communiquer pour satisfaire à ses besoins. Il développe, avec la raison, des stratégies de réussite pour ce qu'il veut. La raison ne peut alors se retirer du contexte. L'acteur est libre, mais n'est pas libre de son environnement. La rationalité, tant dans l'action que dans la communication, doit avoir ses fondements dans le présent, qui est nécessairement historique, aussi bien que dans la relation entre les acteurs. Comme nous l'avons déjà expliqué, l'unicité de cette relation et l'historicité des participants conduisent à une rationalité tout à fait unique. L'acheminement vers un but est sans doute encore l'idée à la base, mais la façon dont l'individu construit des stratégies, la rationalisation, doit aussi être unique au moment de la communication.

On trouve des propos semblables chez Hume ${ }^{18}$. Cependant, ce dernier constate qu'on possède plus que la raison et la passion. Selon lui, ce ne sont pas les seuls éléments qui interviennent dans le comportement humain. Il suggère que l'humain a des inclinaisons pour la compassion et la générosité, qu'il a des façons d'agir qui sont inhérentes à son humanité. D'après Hume, l'humain a des comportements qui relèvent de la nature. Alors, ces comportements intrinsèques influencent-ils ses comportements, ses façons d'agir et de communiquer? L'interprétation de ce qu'est la compassion ou la générosité est-elle universelle? Autrement, si les inclinaisons intrinsèques se plient à l'environnement, on doit constater que les résultats seront tout à fait uniques.

En psychologie, l'axiome utilitariste est utilisé avec une certaine précision. Plusieurs psychologues suggèrent que la cognition - dans son sens spécifique de capacité intellectuelle supérieure - est un élément essentiel dans la prise de décision. Un individu fait des choix pour maximiser ses gains et minimiser ses pertes. Cependant, on doit prendre en considération des éléments qui ne découlent pas de l'inconscient

17 Thomas Hobbes, Leviathan, Cambridge, Cambridge University Press, [1651] 1991.

18 David Hume, A treatise of human nature, Oxford, Clarendon Press, [1740] 1978. 
mais qui relèvent de l'individu. Ses préférences personnelles, ses goûts et même son état émotionnel au moment où il prend une décision sont tous des éléments uniques. Karni ${ }^{19}$ adopte ce point de vue. À la base, on retrouve l'axiome utilitariste. Toutefois, l'auteur suggère certaines modifications, certains ajouts. Il propose qu'on prenne en considération la manière dont l'individu perçoit la vie. Lorsqu'il est mis dans une situation et qu'il doit faire un choix, ses possibilités sont innombrables. Selon Karni, dès le départ la probabilité de chaque option n'est pas équivalente. Certains choix seront préférés par la personne et ces options auront alors une plus grande probabilité d'advenir. Ce chercheur suggère aussi que la façon dont les risques sont perçus influence la probabilité de faire un choix plutôt qu'un autre. Alors comment peut-on faire pour prendre en considération tous les éléments qui agissent sur un individu quelconque et les évaluer tous avec la même perspective qu'a cet individu avant de faire un choix ? Les besoins inconscients de satisfaire aux inclinations intrinsèques pour la compassion et la générosité, ainsi que les préférences conscientes jouent tous un rôle dans la prise de décision, dans la formulation de stratégies ainsi que, finalement, dans le choix de stratégies lui-même.

Sans aller plus en détail, on se rend compte que l'hypothèse utilitariste n'est pas questionnée. Elle joue le rôle d'une constante ; elle est le corollaire d'une autre réalité, soit la cognition. Alors l'omniprésence du raisonnement de l'action humaine est sous-entendue dans cette recherche. Le fait met en évidence le poids que porte cette théorie. Elle est l'élément de base. Sans la certitude de la théorie de départ, la recherche construite n'a pas de fondement.

On remarque le même phénomène dans d'autres études. Dans une recherche menée par Chou et $\mathrm{Chi}^{\prime} \mathrm{n}^{20}$, on suggère que l'abus de drogues, spécifiquement de la marijuana, est surtout influencé par les gains potentiels contre les pertes possibles perçues par les adolescents : plus les adolescents voient la drogue comme étant bénéfique, pour eux,

19 Edi Karni, "Subjective expected utility theory with state-dependant preferences ", Journal of Economic Theory, vol. 60, 1993, p. 428-438.

20 Kee-Lee Chou et James Chi'en, «Utility theory and adolescent drug abusers in Hong Kong ", Child and Adolescent Social Work Joumal, vol. 14, n 6, 1997, p. 397 412. 
plus il est probable qu'ils en consomment. Encore ici, on se rend compte que l'idée selon laquelle on agit en fonction de la maximisation des gains et la minimisation des pertes est bien à l'œuvre. Les résultats sont construits à partir de l'axiome utilitariste, lequel doit être tenu pour acquis pour que les résultats puissent être interprétés.

En sociologie, on peut trouver l'application de l'axiome utilitariste sur des phénomènes sociaux. Selon Muller $e t a l^{21}$, sur la scène politique, la rébellion peut être expliquée en partie par l'intérêt des citoyens. Les auteurs expliquent que la participation à la rébellion est dominée par la croyance que le groupe aura du succès, ce qui fera en sorte que chaque individu en profitera. On remarque comment l'intérêt domine cette théorie.

En économie, on trouve l'hypothèse utilitariste dans son état le plus pur. Les économistes suggèrent tout simplement que les gens agissent dans le but de maximiser leurs gains et de minimiser leurs pertes. Les recherches ne questionnent pas l'hypothèse utilitarisme comme telle ; elles utilisent plutôt l'utilitarisme comme un point de départ. Selon la recherche de Lipman ${ }^{22}$, une personne ne peut appréhender toutes les possibilités avant de prendre une décision en ce qui concerne la façon optimale de servir son intérêt. Même dans une recherche de ce type, le questionnement ne vise pas l'intérêt. Le questionnement vise seulement le processus d'analyse qui produit la décision ; le but de la décision est toujours constant.

Ce sont tous des exemples de la manière dont agit l'axiome utilitariste et de ce qu'il œuvre à l'intérieur d'un cadre théorique spécifique. On pourrait remplir des pages et des pages à force d'illustrations. On verrait chaque fois qu'on surrationalise l'esprit humain pour comprendre ses actions. En le faisant, on croit être en mesure de prédire le comportement. À la limite, si la théorie est bonne, on devrait pouvoir prédire le comportement sans trouver d'anomalies, c'est-à-dire que les gens devraient agir comme la théorie le prédit. Si le but que vise une

21 Edward Muller, Henry A. Dietz et Steven E. Finkel, «Discontent and the expected utility of rebellion: The case of Peru ", The American Political Science Review, vol. 85, no 4, 1991, p. 1261-1282.

22 Barton Lipman, « Decision theory without logical omniscience: Toward an axiomatic framework for bounded tationality », The Review of Economic Studies, vol. $66, \mathrm{n}^{\circ} 2,1999$, p. 339-361. 
personne est indépendant de l'environnement dans lequel elle le formule, si les besoins ne sont pris en considération lorsque l'acteur arrête une stratégie, alors le comportement devrait être uniforme et, par conséquent, on devrait pouvoir le prédire. Cependant, si on trouve des anomalies, cela veut dire que, pour le moins, la théorie n'est pas applicable universellement.

\subsection{Communication}

La communication interpersonnelle fait l'objet de nombreuses études. On rencontre des études sur la communication entre partenaires dans un couple, entre parents et enfants, entre des individus en position d'autorité et leurs subordonnés et entre des individus de différents groupes ethniques. L'un des préceptes des théories auxquelles on recourt normalement veut que, à la source de toute communication interpersonnelle, il y ait une intention. Cette intention est la raison pour laquelle la communication s'active.

La communication entre individus, et surtout à l'âge de la technologie, peut prendre plusieurs formes. Ces formes sont souvent des dérivés du dialogue entre deux individus. Rouselle ${ }^{23}$ fournit un schéma élémentaire pour rendre compte d'un échange d'information entre deux personnes quand il est perçu comme unidirectionnel. Une vision unidirectionnelle de la communication élimine beaucoup trop d'éléments pour être considérée comme la façon dont on communique. On ne parle pas à quelqu'un, on parle avec quelqu'un; et l'échange nécessite une capacité d'ajustement des interlocuteurs. Un regard attentif sur l'acteur qui parle révèle déjà l'influence qu'a le récepteur du message sur la personne qui s'adresse à lui.

Lors d'une transmission d'information vraisemblablement unidirectionnelle, l'acteur A transmet un message à l'acteur B. On ne peut concevoir, dans un tel schéma, l'influence de la réaction de l'individu $\mathrm{B}$, le contexte du message et même l'historicité de chaque participant. L'approche est par trop limitative. La communication ne peut être réduite ainsi, même si le message, lui, le peut. Dans une logique intentionnelle, il faut qu'un agent communicant veuille transmettre de l'information pour que circule de l'information entre lui et les destinataires de ses messages; mais il faut 
bien reconnaitre que, même si cela a été le cas, une fois que la communication a pris forme, elle se développe de façon unique entre les deux participants. Elle est influencée par tellement de facteurs qu'on peut même suggérer la possibilité que l'intention, si elle n'a jamais eu quelque apport, soit perdue au cours du dialogue entre les participants. Selon Laflamme ${ }^{24}$, on trouve des facteurs qui influencent la communication interpersonnelle comme l'historicité des participants, le contexte dans lequel l'échange a lieu aussi bien que la relation entre les participants. Ces facteurs créent une relation unique entre les agents communicants. Les participants communiquent, partagent et échangent des informations de façon singulière puisque chacun est unique.

Si l'intention n'est pas le facteur déterminant dans un échange entre deux interlocuteurs, on doit supposer que cet échange est finalement le résultat de l'interaction entre les participants. Examinons le travail de Girard $^{25}$ dans lequel elle se penche sur le rôle de l'intention dans les échanges libres. Girard suggère d'abord que, si le discours entre les interlocuteurs n'est pas seulement intentionné, alors il ne peut être seulement rationnel. Elle montre que l'échange a un caractère duel : il sollicite aussi bien la raison que l'émotion.

Girard suggère aussi que si l'intentionnalité, et par extension la rationalité, domine l'échange, c'est qu'il ne peut y avoir de position émorationnelle avant l'échange. Ce qu'elle découvre, à l'aide de ses analyses, c'est que les énoncés à l'intérieur des échanges ne peuvent être expliqués à fond à partir d'une perspective pour laquelle l'acteur est strictement rationnel. En d'autres mots, lors de la communication d'un message donné ou d'une information spécifique, l'élimination de l'influence pré-communicationnelle est impossible et donne lieu à une position avant l'échange qui n'est pas strictement rationnelle et, donc, qui n'est pas réductible à l'intention.

Si l'intentionnalité ne précède pas nécessairement l'échange, d'où viennent les propos entre les interlocuteurs? Girard découvre que les propos se veulent surtout le résultat de l'interaction entre les interlocuteurs. S'il y a une intention, et alors le discours est rationnel, c'est qu'il ne peut se modifier. Ce n'est pas le cas. Elle rencontre des échanges mouvants,

24 Simon Laflamme, Communication et émotion..., op. cit.

25 Mélanie Girard, Relations bumaines et production d'information..., op. cit. 
ce qui suggère que la rationalité ne peut être le facteur dominant ou plutôt qu'elle n'est certainement pas le seul élément déterminant de la circulation de l'information. Si c'est le cas, l'échange doit alors être compris à l'aide d'autres facteurs.

L'agir intentionnel, dans un discours, doit donner lieu à une certaine rigidité des échanges. C'est donc dire que si la volonté d'accomplir une mission quelconque se trouve être le facteur déterminant de l'échange, on doit être capable de retrouver cette volonté. Cette volonté se montrerait sous forme de rigidité dans le discours; on devrait en trouver la trace dans une impossibilité de s'éloigner de la mission. Comme Girard le montre clairement, si on peut expliquer un échange à partir d'une intention, c'est que le discours est immuable. L'échange ne peut pas montrer de flexibilité s'il n'est qu'une manifestation d'une volonté mise en cuvre sans considération extérieure. Ce manque de considération donne cours à des échanges difficiles ou les rend même impossibles. On peut alors croire que des interlocuteurs chargés d'accomplir une mission peuvent enfin se trouver dans l'impossibilité de communiquer efficacement. En l'absence de flexibilité, l'écoute d'une réponse d'un autre interlocuteur et l'incapacité de modifier son propos peuvent rendre l'échange difficile et, à la limite, impossible.

Girard observe que les discours se transforment au cours de l'échange. Elle fait état du caractère à la fois rationnel et émotif des échanges. À partir de son ouvrage, on peut constater que les agents communicants réagissent à l'information qui est échangée. L'assimilation de cette information et sa valeur sont différentes selon le point de vue de chaque interlocuteur. On ne peut croire que deux personnes qui sont informées au sujet d'un événement peuvent avoir la même réaction. Par exemple, toutes les personnes n'auront pas la même réaction en apprenant la découverte d'une vache folle dans un troupeau d'animaux. Les réactions à des énoncés sont donc en partie des points de vue qu'on peut relier à des valeurs, qui, elles, ont un caractère duel de rationalité et d'émotivité.

On peut alors se questionner au sujet de l'universalité de l'axiome utilitariste. Le principe est logique. Cette théorie a une simplicité attrayante. Sans trop la questionner, on pourrait se laisser séduire par sa simplicité. L'acteur est mené par des passions et des désirs. Ceux-ci doivent trouver satisfaction. L'acteur utilise alors l'habileté à la planification ou à la construction de stratégies. Ces stratégies mènent à la réalisation de 
désirs. Le tout semble cohérent. Cependant, si on se limite simplement à cette dimension, on remarque certains paradoxes dans le comportement humain. Le don est l'un des ces paradoxes. Comment peut-on expliquer le fait de donner pour satisfaire à ses désirs dans le don d'organe ? Ou comment expliquer le don de sang? Ou encore comment expliquer les dons de charité anonymes? L'interprétation utilitariste alors, bien que souvent évoquée pour rendre compte de tels phénomènes, scmble alors un peu tirée par les cheveux. Toutes ces questions font en sorte qu'on peut s'interroger sur l'universalité de l'axiome utilitariste. Cette théorie donne des éléments qui peuvent servir à l'élaboration d'hypothèses.

Les concepts de la rationalité et de l'intentionnalité sont liés de façon inséparable dans les ensembles théoriques, comme le montrent Girard, Laflamme et Roggero : «Dans ces cadres théoriques, si l'acteur peut être déclaré comme rationnel, et de façon principielle, c'est aussi qu'il est simultanément intéressé, stratégique, conscient, réfléchi, autonome, intentionné $»^{26}$. Cependant, le lien entre l'intention et la rationalité ouvre la porte à un dilemme. Une fois engagé, l'acteur social intentionné peut difficilement s'adapter aux demandes extérieures qui ne sont pas reliées à son intention. Mais l'acteur social communique aisément, sans difficultés. Il est capable d'écouter, d'interpréter et de faire avancer l'échange. Il peut s'adapte et montre une flexibilité à l'intérieur d'un échange dynamique. Cette capacité d'ajustement s'explique difficilement à partir de la perspective d'un acteur social purement rationnel. De plus, l'intention à l'intérieur d'un discours rationnel devrait être repérable. Si ce n'est pas le cas et que l'échange se montre dynamique, fluide et mouvant, c'est que d'autres éléments doivent intervenir dans les échanges et doivent être inclus dans l'analyse pour en comprendre les fondements.

\section{Hypothèses}

Le but de la recherche est de vérifier si l'intention dans l'information qui circule entre les agents communicants se manifeste aussi essentiellement que le veulent les théories de l'action. Ces propos débouchent sur trois hypothèses principales. En premier lieu, si l'axiome utilitariste est applicable

26 Mélanie Girard et al., " L'intention est-elle si universelle que le prétendent les théories de l'action?", op. cit., p. 115. 
en ce qui concerne la communication, le but de la communication est de transmettre un message qui mène vers un but. Toute communication, même celle qui à lieu entre étrangers, doit être intentionnée : c'est pour s'approcher de ce qu'on veut qu'on communique. Dans ce cas, l'intention est une partie importante du discours et on devrait être capable de la trouver.

Toutefois, si l'intention ne domine pas la communication et qu'elle constitue seulement un élément parmi plusieurs autres, alors un échange intentionné sera plus difficile à trouver. L'intention, puisqu'elle est une partie du discours, doit alors, dans ces circonstances, faire partie d'un réseau complexe. Cela soulève plusieurs questions. Quand l'intention prend-elle forme? Avant ou après le début de l'échange? Si c'est durant l'échange, à quel moment durant le discours? Ce sont là des difficultés qu'on rencontre si l'intention ne mène pas le discours.

En deuxième lieu, dans le cas où l'intention d'un participant précéderait l'échange, on pourrait s'attendre à un discours clairement intentionné. Si on est assuré qu'il y a une intention qui précède l'échange, alors cette intention devrait dominer l'échange et être facilement observable. Mais si ce n'est pas le cas, on pourrait s'attendre à une mouvance dans l'échange. L'intention est mêlée à d'autres composantes et, alors, il devient le discours entre les participants.

En troisième lieu, enfin, lorsque les deux participants sont intentionnés antérieurement à l'échange, on peut s'attendre à deux possibilités. D'abord, si l'intention est le seul élément qui domine la communication, alors deux individus intentionnés rendraient la communication difficile et, à la limite, impossible. Cependant, si l'intention ne domine pas la communication, on pourrait s'attendre à ce que le participant fasse preuve d'une capacité d'ajustement. La communication ne serait pas strictement dominée uniquement par l'intention.

\section{Méthode}

Nous tentons de manipuler l'intention, soit la présence explicite d'une intention dans un discours. Dans ce projet, l'intention peut se définir comme étant une représentation mentale qui cherche à être mise en action. Notre intention, pour jouer sur les mots, est de vérifier si l'intention, chez des agents communicants, joue le rôle que prévoient les théories pour lesquelles cette intention est au principe de tout faire et de 
tout dire humain ; nous pensons, entre autres, aux théories de l'action. Pour enfin en arriver à manipuler l'intention, les groupes ont été limités à deux interlocuteurs et deux missions ont été choisies.

Notre échantillon est composé de 60 étudiants de l'Université Laurentienne et du Collège Boréal. Les échanges ont eu lieu en laboratoire. Les participants étaient groupés par deux, ce qui a permis 30 échanges divisés selon trois groupes expérimentaux. La collecte a débuté en février 2004 et s'est terminée en octobre 2004.

Deux thèmes ont été choisis par l'expérimentateur, indépendant des sujets : celui de l'augmentation des frais de scolarité au niveau postsecondaire et celui de la double cohorte ${ }^{27}$. Puisque l'échantillon est tiré d'une population étudiante au postsecondaire, ces thèmes étaient forcément pertinents. Avant de procéder avec l'échange, le chercheur s'assurait que les participants comprenaient la signification des termes.

Dans les dyades de la première condition, les individus étaient libres de discuter de l'augmentation des coûts au niveau postsecondaire. Dans les dyades de la deuxième condition, un sujet recevait une mission qu'il devait remplir, soit celle d'informer son interlocuteur des bienfaits des augmentations des frais de scolarité. Cette instruction était donnée à l'insu du deuxième participant. Par la suite, les consignes générales, celles qui avaient été données au premier groupe, étaient livrées au deuxième groupe. Dans les dyades de la troisième condition, les deux sujets avaient une mission à remplir. Le premier participant devait tenter d'informer l'autre interlocuteur des bienfaits des augmentations des frais alors que le second devait tenter d'informer son interlocuteur des effets néfastes de la double cohorte. Chaque directive était donnée au participant à l'insu de l'autre. Le chercheur demandait à chaque participant s'il se sentait en mesure d'accepter la mission. Une fois que les directives étaient données et que la mission était acceptée, une consigne générale était lue qui indiquait que les participants pouvaient discuter des frais de scolarité au niveau postsecondaire et des effets de la double cohorte.

27 La double cohorte se rapporte à l'entrée de deux cohortes d'étudiants au postsecondaire lorsque la dernière année du secondaire (CPO ou 13" année) a été abolie par le ministère de l'Éducation de l'Ontario. En cette année, les finissants de la $12^{c}$ année et du CPO se sont trouvés simultanément au postsecondaire. 
La session débutait avec une brève explication de ce qu'on allait faire. Par la suite, les participants devaient signer un formulaire de consentement indiquant leur acceptation de collaborer. Les séances étaient enregistrées sur vidéocassettes. À un moment donné, les deux participants étaient interrompus par le chercheur, ce qui signifiait la fin de l'échange. La durée d'un échange typique était d'environ vingt minutes. À la suite de l'échange, il y avait une courte discussion avec chaque dyade sur les objectifs de la recherche.

Chaque séance fut transcrite, puis chaque échange fut identifié par un chiffre suivi d'une lettre, soit $\mathrm{A}, \mathrm{B}$ ou $\mathrm{C}$. Ces lettres indiquent la condition de l'échange. " $A$ » correspond aux échanges libres, « $B$ » aux échanges où un seul participant avait une mission et « $C$ » aux échanges où les deux participants avaient une mission. Un tableau fut construit pour faciliter l'analyse des discours.

Dans la première condition nous cherchions :

- La part du discours qui ne peut être attribuable qu'à l'échange luimême.

- Une intention qui naît de l'échange :

1. la cause de cette intention;

2. la persistance ou non de cette intention.

- Une intention qui précéderait l'échange.

Dans la deuxième condition (où l'on donne une mission à un sujet seulement), nous cherchions :

- La capacité de maintenir le propos selon la tâche :

1. les propos correspondant à la tâche ;

2. les propos éloignés de la tâche.

- La fréquence des énoncés :

1. de la part du sujet $A$ qui correspondent à cette tâche ;

2. de la part du sujet $\mathrm{B}$ qui correspondent à cette tâche.

- La part du discours qui ne peut être attribuable qu'à l'échange luimême.

Dans la troisième condition (où chaque sujet a une mission à accomplir), nous cherchions :

- La capacité de maintenir le propos selon la tâche :

1. les propos correspondant à la tâche ;

2. les propos éloignés de la tâche.

- La fréquence des énoncés :

1. de la part du sujet $A$ qui correspondent à cette tâche ;

2. de la part du sujet B qui correspondent à cette tâche.

- La part du discours qui ne peut être attribuable qu'à l'échange. 


\section{Analyses de données}

On aura compris que le but de cette recherche est de vérifier si la communication verbale entre deux interlocuteurs résulte d'une intention précommunicationelle et surtout, si elle est un propos unique entre deux individus. Il s'agit donc de comprendre en quoi l'échange est attaché au contexte et comment il dépend des participants et de la relation même qu'entretiennent entre eux les interlocuteurs. Il s'agit aussi de découvrir quels facteurs font partie de l'équation et dans quelle mesure ils sont exclusifs les uns des autres.

\subsection{Rigidité ou fluidité}

Comment peut-on s'assurer de la fluidité de l'échange et de sa nature relationnelle et, par ricochet, de son caractère émorationel ? On peut chercher si certains interlocuteurs ont pu changer de point de vue sur un des sujets abordés. Si c'est le cas, on doit alors croire que le changement vient d'une réaction à l'information donnée par l'interlocuteur plutôt que d'être la conséquence d'une intention antérieure. Ce changement est indicatif d'une transformation ainsi que d'une fluidité de l'échange. Ce phénomène est une démonstration de la souplesse de l'échange, ce qui rend peu probable l'hypothèse d'un discours défini par une conscience rationnelle-intentionnelle.

On peut aussi imaginer que, dans les discours intentionnés, auxquels correspondent les deuxième et troisième conditions, on devra noter une persistance de l'intention tout au long du discours. Si on ne parvient pas à trouver cette persistance, il doit y avoir une transformation de l'intention initiale, ce qui témoignerait encore d'une transformation. Dans ce cas, à nouveau, une modélisation rationalisante invite à la suspicion.

\subsection{Portrait d'un échange}

Lorsqu'on examine les échanges dans leur globalité, on remarque qu'il n'y a pas de différence significative entre les conditions.

On remarque une uniformité remarquable des tours de parole entre les conditions. On trouve 191 tours de parole dans la première condition, 180 dans la deuxième et 192 dans la troisième. De plus, on ne remarque pas de différence du nombre de répétitions de propos d'une condition à l'autre. Parmi les conditions, le nombre de répétitions 
varie de 18 à 23 . On ne remarque pas de différence évidente du nombre d'énoncés qui naissent de l'échange. On observera 149 énoncés qui naissent de l'échange dans la première condition, 129 dans la deuxième condition et 120 dans la troisième condition.

\begin{tabular}{|l|c|c|c|}
\hline \multicolumn{4}{|c|}{ Tableau 1 : Classification de propos } \\
\hline & Condition 1 & Condition 2 & Condition 3 \\
\hline Énoncés reliés aux frais de scolarité & 48 & 47 & 30 \\
\hline Énoncés reliés à la double cohorte & 0 & 0 & 21 \\
\hline Énoncés non reliés à une intention & 124 & 125 & 132 \\
\hline Assentiments & 52 & 48 & 57 \\
\hline Conséquences & 14 & 17 & 22 \\
\hline Silences & 6 & 5 & 4 \\
\hline Tours de paroles & 191 & 180 & 192 \\
\hline Répétitions & 18 & 23 & 23 \\
\hline
\end{tabular}

Quelle que soit la condition, on observe une similitude remarquable des arguments utilisés au cours des échanges. Quelquefois, les arguments sont énumérés un après l'autre. À d'autres moments, ils sont éparpillés tout au long du discours. Parfois, ils sont peu repérables. Quand les arguments sont épuisés, les échanges prennent souvent en défaut l'intention initiale, c'est-à-dire que l'échange devient difficilement prévisible à partir de ce moment. On constate un silence, une répétition, un changement de point de vue chez un interlocuteur. Quelqu'un se met à raconter une histoire ou à présenter un sujet d'intérêt personnel.

Le discours devient immédiatement imprévisible. Même si les arguments se ressemblent d'un échange à un autre, la façon dont chaque interlocuteur arrive à en discuter et à y faire référence est impossible à prédire. Ce phénomène est le point de départ de notre analyse.

\subsection{Première condition : un cas ouvert}

Dans la première condition, où il n'y avait pas de missions explicites, on observe que la grande partie des tours de paroles ne peut être expliquée que par la dynamique de l'échange. Le contenu de ce qui est dit provient 
non d'une directive, mais du contexte de l'échange. Il y a une mouvance de l'échange, laquelle crée des imprévus. Ces imprévus sont parfois utilisés par l'interlocuteur comme point de lancement ou de repère pour faire avancer l'échange. L'imprévisibilité d'un énoncé vient souvent d'un référent personnel, un vécu que les interlocuteurs ne partagent pas et qui, par conséquent, ne peut être prédit puisque son existence n'est pas connue préalablement à la déclaration d'un des interlocuteurs. On observe des répétitions de propos non dans le but de communiquer de nouvelles informations, mais surtout pour souligner le propos d'un locuteur. On remarque aussi de l'information d'ordre personnel, dans le cas où les interlocuteurs se connaittraient, des activités auxquelles eux seuls ont participé et dont ils peuvent discuter - ce qui serait quasiimpossible de reconstruire chez des interlocuteurs étrangers - et qui atteste du dynamisme de l'échange. L'échange se perpétue à partir de ses antécédents propres, construits au cours de l'échange, et qui sont composés de plus d'une position précommunicationnelle. Les imprévus, c'est-à-dire les réponses d'un interlocuteur, deviennent les portes par lesquels les échanges peuvent diverger de leurs directions initiales. Prenons un exemple (tiré de la conversation PJ-03A, tours de parole numéros 157 à 176) :

157. Répondant $A: A h$ ! il va falloir faire de quoi là.

158. Répondant B : Oui !

159. Répondant A : Toutes les girls ensemble là.

160. Répondant B : Uhuh ! Oui. C'est drôle, parce que (nom propre) c'est une sœur, puis tu penserais jamais qu'est une soeur. (Inaudible.) Elle est fine.

161. Répondant $A$ : Elle enseigne?

162. Répondant B : Bien (inaudible). Oui.

165. Répondant $\mathrm{A}: \mathrm{J}$ 'ai hâte d'être rendue là pour pas être encore prise (inaudible).

166. Répondant B : Comme dans le milieu social, c'est pas pire. Ça dépend quelle option que t'es, parce que je sais que l'option $\mathrm{X}$ c'est comme party all the time.

167. Répondant $A$ : Ah oui!

168. Répondant B : Ah oui, c'est party, party party, party, party. Puis c'est comme nah. J'ai été à une couple de petites choses là, mais pas rien de... j'étais pour dire qu'il y a du feedback, c'est juste... 


\section{NPSS, VOLUME 2, NUMÉRO 1, 2006}

169. Répondant $A$ : Hahahaha.

170. Répondant B : C'est drôle la façon que ça sonnait. Mais c'est quand même, ils sortent toujours comme à soir comme il y a beaucoup de gens qui ont pas de cours le matin. So, souvent ils sortent à soir ils vont jouer au poker ou ils vont faire plein de chose ou...

171. Répondant $\mathrm{A}:$ Hum.

172. Répondant B : Puis aussi c'est cliqué aussi. Tu as tes petites cliques.

173. Répondant $\mathrm{A}: \mathrm{Ah}$ ! Ça, j'aime pas ça.

174. Répondant B : C'est comme, c'est comme si tu es a... eh... au secondaire ; des fois, c'est comme ça que tu te sens. Comme, moi, je m'en fous bien, là. C'est comme même (inaudible) non sorry comme. Moi j'ai un jeune, je peux pas sortir deux, trois fois par semaine, comme puis partyer puis dépenser de l'argent là-dessus.

175. Répondant $A:$ C'est ça.

176. Répondant $\mathrm{B}:$ Je suis pas dans la même ligue qu'eux autres. Puis ils sont chiâleux.

En examinant cet enchaînement de tours de paroles, spécifiquement les énoncés 157 à 159, on remarque une amitié entre les répondantes. On détecte une familiarité et une amitié non seulement dans le propos, les sorties, mais aussi dans la tonalité et dans le rythme. Ces indicateurs suggèrent une absence de formalité et rappellent qu'elles se connaissent. Les participantes ne suivent plus les directives qui leur demandent de parler de l'augmentation des frais de scolarité au postsecondaire et discutent d'une sortie sociale puisqu'elles se connaissent. Cependant, elles ne savaient pas qui était leur partenaire avant le moment de l'échange. On ne peut pas croire que la mission d'inviter quelqu'un à une sortie sociale était préméditée. Si, en fait, c'était le cas, l'identité du participant n'aurait pas eu d'influence et il y aurait eu l'invitation à la sortie quel que soit l'interlocuteur. Cette éventualité est difficilement acceptable. Un tel comportement serait peu probable. Ce sont surtout les circonstances de la situation qui ont permis à l'interlocutrice de faire une invitation au cours de l'échange. On ne peut attribuer ces circonstances à l'intention d'un participant ; il faut les rapporter au contexte, au hasard qui a rendu l'acte possible. Dans ce cas, l'intention a été modifiée, transformée pour satisfaire au contexte. 
La première remarque annonce le sujet de la discussion. On ne peut trouver de liens directs avec la consigne initiale. La conversation s'est déplacée des thèmes des frais de scolarité vers l'expérience vécue en commun à l'école X. Cette transformation ne devait être possible que pour faire avancer la discussion sur le sujet initial, si on l'interprète à l'intérieur d'un modèle rationnel-intentionnel. Les énoncés doivent faire avancer l'intention initiale. Ce n'est pas le cas. Le sujet de la discussion est changé et on ne peut faire de raisonnement rationnel qui relierait ces énoncés au sujet de départ. L'échange est dynamique et propre à ces deux interlocuteurs.

Cependant, les tours de paroles $168,170,172,174$ et 176 semblent tous avoir un lien entre eux. L'agent communicant « $\mathrm{B}$ » informe l'agent "A », en exprimant, au passage, son opinion sur les comportements des élèves de l'école $\mathrm{X}$ qui sont dans l'option X. Si, enfin, c'est bien l'intention du répondant « $\mathrm{B}$ », où se trouve l'intention dans les énoncés antérieurs, spécifiquement lors de l'invitation? L'invitation sociale qui a appelé à l'information sur les comportements de certains élèves n'était pas prévisible. La réponse affirmative ne peut être seulement rationnelle. Elle doit faire appel à des expériences antérieures, au plaisir d'être avec cette personne. Cette réponse a forcément des assises rationnelles et émotionnelles.

La possibilité de faire une sortie commune ouvre la porte à l'évocation, chez l'une des participantes, de l'expérience de l'école X. Cette porte permet à la participante de parler de ses frustrations et de ses impressions, de son expérience. On ne peut concevoir que cette ouverture fait partie d'un projet, qu'elle soit strictement intentionnée ou rationnelle. Les participants doivent interpréter cette ouverture pour ensuite se donner la réplique. Puisque la réplique n'a pu être déterminée à l'avance, elle ne fait pas partie du projet initial ; donc elle ne relève pas d'une intention originelle. Si la participante répond au propos, comme c'est le cas, c'est qu'il y a eu une transformation de l'intention initiale, ce qui serait impossible dans la perspective rationnelle.

Cet exemple, aussi bien que n'importe quel autre qu'on retrouve dans cette condition, témoigne à l'évidence d'une dynamique entre les participants. On retrouve des propos qui sont interprétés par l'interlocuteur, lequel exprime ensuite une opinion, pour finalement faire écho au propos. Cette interprétation unique, qui a eu lieu dans un contexte unique, ne peut être le fruit d'une intention. Elle doit être le 
résultat de l'interaction des nombreuses variables dont est composé l'échange. Certes, un interlocuteur peut avoir une intention, mais cette intention doit faire partie d'un réseau de variables; il ne peut constituer un facteur unique ou ultime de détermination.

\subsubsection{Un échange intentionné ou plutôt émorationnel}

Lorsqu'on examine de plus près les énoncés des participantes, on constate, au moins en partie, que leur familiarité leur a permis de discuter d'une sortie sociale, puis de faire une évaluation subjective de la majorité des étudiants qui se trouvent dans l'option $\mathrm{X}$. On doit maintenant se poser la question suivante : parle-t-on d'intention dans ce cas-ci et, si oui, d'où vient cette intention ? D'abord, en regardant la totalité de l'échange, on remarque que la participante utilise son expérience à l'école $\mathrm{X}$ comme point de départ pour exprimer plusieurs de ses idées. Par les réponses de la participante « $\mathrm{B} »$, on se rend compte que cette dernière n'a pas d'expérience avec l'école $\mathrm{X}$; que cette expérience se rapporte plutôt à l'université de façon plus générale. Maintenant on doit se poser la question de savoir si, puisqu'une des participantes n'est pas familière avec les référents de l'autre, c'est la façon la plus efficace de transmettre son point de vue. En d'autres mots, puisque l'université est nécessaire pour aller à l'école $\mathrm{X}$ et, par conséquent, que les deux participantes ont de l'expérience universitaire, alors pourquoi choisir un référent obscur? Est-ce la meilleure façon de faire partager son point de vue ? Si la réponse est négative et que la raison pour laquelle il est fait référence à l'école $\mathrm{X}$ est dictée par la familiarité ou même par besoin de la participante " $A$ » de prendre position et d'agir sur l'échange en s'assurant d'utiliser une référence commune pour ensuite faire allusion à des connaissances que « $B$ » n'a pas, alors on doit supposer que l'intention n'est pas vraiment dominante dans ce qui a été observé.

\subsubsection{Une intention qui précéderait l'échange}

" $\mathrm{A}$ » a introduit la référence à ceux qui sont dans l'option $\mathrm{X}$ par l'invitation qu'elle a faite à son amie. Le point de départ a été les pratiques sociales d'une enseignante qui a été enfin reliée aux pratiques du groupe de l'option X. Puisque la porte a été ouverte par la participante « $\mathrm{B}$ », peut-on supposer que la participante « $\mathrm{A}$ » avait 
manipulé l'échange pour s'assurer d'une invitation qu'elle pourrait ensuite utiliser comme point de départ pour verbaliser son opinion sur les étudiants de l'option X ? Ou n'est-il pas plus probable que la participante " $A$ », avec ses connaissances et son expérience, ait saisi l'occasion pour discuter d'un phénomène, qui est relié à la sortie, pour convaincre l'autre des habitudes des élèves de l'option $X$ ? Si l'intention domine et que le but de cet échange est de convaincre l'autre interlocutrice, on doit se poser la question : De quoi « A » veut-elle convaincre « $\mathrm{B} »$ ? $\mathrm{Si}$ on ne peut efficacement répondre, on doit conclure que l'intention de « $\mathrm{A} »$ n'est pas totalement claire et que, par conséquent, elle ne peut constituer un facteur dominant. On voit plutôt qu'il y a plusieurs possibilités d'intention sans, en fait, qu'on puisse relever une cause dominante. On peut suggérer que " $A$ " veut communiquer l'aspect négatif de l'option $\mathrm{X}$ et ainsi décourager « $\mathrm{B}$ » d'aller dans cette direction. Pour voir ainsi les choses, on doit supposer que « $\mathrm{B} »$ veut entreprendre une formation à l'école $\mathrm{X}$ dans l'option $\mathrm{X}$. Si ce n'est pas le cas, alors cela ne peut être le but ni l'intention de « $A$ ». Supposons maintenant que « $A$ » veut convaincre « $B$ » que, même si elle fait partie de l'option $\mathrm{X}$, elle ne participe pas de façon irresponsable aux sorties sociales. Si cela est le cas, pourquoi soulèverait-elle le sujet? Évidemment, « $\mathrm{B}$ » ne connait pas les habitudes de ce groupe et, donc, n'a aucune raison de croire que « $A$ » ne prend pas ses responsabilités au sérieux et qu'elle n'a pas besoin d'être convaincue.

On pourrait continuer à suggérer des possibilités d'intentionnalité chez « $A$ », mais on se rend compte que les suggestions ne sont que des hypothèses qui invitent, pour le moins, à la suspicion. Dans ce cas, l'intention n'est pas claire. Ni la rationalisation de « $A$ », ni le motif de l'intention de « $A$ » ne peuvent être clairement interprété comme tel, ce qui indique que la rationalité ne définit pas l'échange d'une manière essentielle ou absolue. La dynamique devient tellement complexe dans cet échange qu'on ne peut saisir complètement ce que «A » voulait accomplir.

\subsection{Deuxième condition : une mission}

Dans le cas de la deuxième condition, l'un des participants reçoit une mission explicite : il doit renseigner son interlocuteur sur les bienfaits des augmentations des frais de scolarité au niveau postsecondaire. 
Ce qu'on remarque en premier lieu, c'est qu'il n'y a pas de différence significative entre les conditions 1,2 , et 3 en ce qui à trait aux énoncés reliés aux frais de scolarité. Les échanges qui ont une directive ne contiennent pas plus d'énoncés reliés au thème où à la directive que l'échange où les deux interlocuteurs sont libres. Cela indique-t-il que la directive est ignorée ? Ce n'est pas le cas. On remarque que même s'il n'y a pas de différence statistiquement significative, le participant qu'on a investi d'une mission cherche à l'accomplir. Alors qu'arrive-t-il ? L'interlocuteur s'éloigne de sa mission et de son intention initiale. L'échange avance et se modifie à partir des opportunités qui se présentent. Au cours de l'échange, surtout lorsqu'un interlocuteur tente de répondre à l'autre, une multitude de possibilités se présente. La réponse peut faire avancer l'échange dans sa direction originale ou elle peut le réorienter. Dans ces deux cas, on remarque la mouvance du discours. Il n'est jamais trop rigide pour nuire à l'échange ou jamais trop éloigné pour ne pas trouver de référents dans ses énoncés. Des questions cherchent les réponses à des inconnues. La façon dont ces inconnues vont relancer l'échange n'est pas prévisible et mène à un échange dynamique. Utilisons un exemple concret pour fin de démonstration (tiré de l'échange PJ-14, tours de paroles 1 à 12) :

1. Répondant $\mathrm{A}:$ : Okay, so tu trouves ça déjà assez cher ou...?

2. Répondant $\mathrm{B}:$ Je ne sais pas, j'ai assez d'argent cette année...

3. Répondant $A: S o$ c'est pas trop pire?

4. Répondant $\mathrm{B}:$ Mais je sais que beaucoup de mes amis n'ont pas assez d'argent, comme je le sais pas... peut-être, peut-être c'est trop cher pour ceux qui hum... comme n'ont pas des parents, qui n'ont pas de l'argent, qui doivent faire...

5. Répondant A : Hum... ceux qui prennent de l'OSAP, des choses comme ça...

6. Répondant B : Oui.

7. Répondant $\mathrm{A}$ : Mais si ça montait à 3000 l'année prochaine, tu penserais-tu que ça serait trop ou?

8. Répondant B : Oui.

9. Répondant $\mathrm{A}$ : Comme tu pense-tu que c'est une bonne chose que...

10. Répondant $B:$ Non. 
11. Répondant A : Comme moi, moi je pense que c'est une bonne chose que les frais de tuition montent juste parce que c'est comme ça que toutes les autres choses se font payer comme les sports puis ee... les activités...

12. Répondant $B$ : Okay, mais les équipes de sports devraient faire des prélèvements de fonds.

L'échange débute avec un questionnement. Intentionnellement, l'interlocuteur qui a une mission - l'agent communicant « $A$ »-cherche à y donner cours. On doit alors croire que le questionnement est en fait une méthode pour faire avancer son but. Mais n'est-ce pas plus probable que le questionnement se fait dans le but de recueillir de l'information pour déterminer le point de vue de l'autre participant? Si c'est le cas, on doit croire que cette information sera utilisée par «A » pour définir la méthode à laquelle il recourra pour informer son interlocuteur sur les bienfaits des augmentations. À ce point de la conversation, la mission n'est pas encore rigide. Sa forme doit être déterminée à partir de la réponse de « $\mathrm{B} »$. « $A$ » doit réagir à la réponse de « $B$ » et, comme l'a démontré Girard ${ }^{28}$, la réaction ne peut être que partiellement rationnelle.

Le troisième tour de parole suggère une interprétation de la part de " $A$ » qui pose une question fermée, ce qui n'autorise qu'une réponse par oui ou par non, qui est par la suite contournée par la répondante « $\mathrm{B}$ ». Si cette question a pour but de faire avancer les choses et qu'elle est par la suite contournée, "A » ne doit-il pas modifier son discours ? L'échange continue et on ne peut remarquer aucune difficulté dans l'échange. Les participants continuent leur discussion sans difficulté, ce qui doit être interprété comme une manifestation de la souplesse de la discussion. La fluidité de l'échange rend difficile la notion d'intention pour rendre compte de cet échange.

$\mathrm{Si}$ on continue avec le tour de parole numéro 5 , on remarque une réponse au propos précédent de " $\mathrm{B}$ ». Si le discours de « $\mathrm{A}$ » est intentionnel, c'est que sa réponse doit être intentionnée. Alors pour répondre au propos de « $B$ » sans modifier son discours, « $A$ » a dû prédire le propos de «B » pour l'incorporer dans son discours intentionné. Sinon, il a dû y avoir une transformation du discours, laquelle, finalement, serait indicative d'une mouvance de l'échange, difficilement explicable par la notion d'intention. 
Enfin, on a le tour de parole numéro 11 ; il se compose de deux parties. En premier, " $A$ » affirme son opinion et, ensuite, la fait suivre d'un raisonnement. Peut-on croire que c'est dans le but d'empêcher la progression de sa mission? « $\mathrm{B}$ », dans ses propos antérieurs, a déjà indiqué la possibilité que les frais de scolarité deviennent trop chers et que, par conséquent, ils aient un effet néfaste sur la population étudiante.

Pris individuellement, les tours de parole de « $A$ » ne sont pas indicatifs d'un discours dominé par l'intention. On a pu établir un raisonnement clair ou une rationalité spécifique du discours. Cela est représentatif de ce qu'on observe dans cette condition. On n'a pas pu établir une rationalité qui pouvait relier tous les énoncés à la mission initiale. On a observé des échanges qui se sont transformés à mesure que le discours avançait, les échanges se produisant à partir de la dynamique entre les deux interlocuteurs.

Il semble que l'intention initiale fait partie, sous une forme ou un autre, de la discussion. Le sujet demeure relié aux frais de scolarité et à leur augmentation, mais il a été modifié, changé à partir des demandes du moment.

\subsubsection{Un échange intentionné ou émorationnel}

L'échange qu'on a ici ainsi que tous les autres échanges de cette condition, ne se montrent pas purement intentionnels. L'échange est fluide et, pour que cette fluidité soit possible, les participants doivent ajuster leur discours en fonction de ce qui a été dit antérieurement. Pour ne pas s'éloigner analytiquement de la notion d'intention, on doit croire que toutes les réponses et réactions d'un participant vis-à-vis de ce que l'autre interlocuteur dit doivent être formulées rationnellement. Mais, comme cela a déjà été démontré, on ne peut attribuer toutes les réactions à une rationalisation spécifique.

Dans les dyades de cette deuxième condition, un interlocuteur avait une mission clairement définie. Mais on n'a pas trouvé la marque dominante de cette intention dans l'échange. En moyenne, seulement 47 énoncés ont pu être reliés à la mission initiale. Cela représente environ $25 \%$ des énoncés d'un échange typique. Ce qu'on observe, ce sont des échanges fluides, constitués d'énoncés qui suscitent des réactions de la part des participants, des réactions qui ne peuvent s'expliquer de manière strictement rationnelle. On observe, en effet, des indices de la présence d'émotions, ce qui ne peut exister à l'intérieur 
d'un discours purement intentionné. On peut prendre des exemples de rires, de silences ou de répétition et se poser la question de leur rôle dans l'échange, de la cause de leur apparition et de leur fonction. Ce qu'on constate, c'est que ces phénomènes ne peuvent être tous définis comme faisant partie d'une intention générale. Il doit y avoir des composantes émotives, contextuelles et sociales. Sans ces phénomènes, on ne peut pas expliquer la formation des énoncés de l'échange. Il est vrai que même lorsqu'on prend en considération tous ces éléments, on ne parvient pas encore à prédire le déroulement de l'échange, mais cette lacune peut être expliquée par le caractère émorationnel de l'échange. Une vision relationnelle de l'échange permet de comprendre les changements à partir de la perspective de chaque interlocuteur. Le moment, les personnes et le contexte sont uniques, ce qui rend l'échange unique et, de la même façon, imprévisible.

\subsubsection{Une intention qui précède l'échange}

Au cours de cet échange, plusieurs questions sont posées. Certaines des réponses sont des affirmations, d'autres des négations, d'autres encore sont l'expression d'une opinion ou même des formulations qui se terminent par des questions. Mais la mission de départ est d'informer le partenaire des bienfaits des augmentations des frais de scolarité. Toutefois, on n'arrive pas à trouver cette intention dans sa forme pure. On trouve plutôt des énoncés reliés d'une façon indirecte à la mission originale. Ce fait suggère que l'intention initiale a été modifiée d'une façon ou d'une autre. Mais où se trouve cette modification?

La complexité de la réponse est considérable. Interpréter l'échange à partir de sa structure émorationnelle permet d'entrevoir des réponses. Il y a un échange d'information. Mais cette information n'est pas sans référents et ces référents sont d'ordre personnel et sont formés à partir d'un vécu que les deux participants ne partagent pas de façon identique. Alors l'information échangée doit être interprétée par chaque participant et chaque interprétation est unique. Le contexte dans lequel l'échange a lieu est aussi unique. C'est un moment spécifique dans le temps, qui est accompagné par des demandes contextuelles de ce moment. Pris ensemble, tous ces éléments aident à former le discours. Dans cet ensemble, les variables interagissent pour donner cours à un échange. Ces variables, prises une à une, ne permettent pas de comprendre comment la communication se produit. 
On n'arrive pas à relier les énoncés de cette condition à la mission initiale. Dans la grande majorité des cas, les énoncés sont le fruit de l'échange plutôt que d'une rationalisation antécédente, c'est-à-dire que les propos ne sont pas prévisibles. Ils apparaissent à mesure que l'échange avance et que chaque interlocuteur interprète l'information qui lui est présentée. Les propos renvoient aux frais de scolarité ou ils ont pour cause l'échange lui-même. Encore une fois, l'interlocuteur, même chargé de sa mission, n'a pu concentrer les propos autour du thème qu'il avait à aborder à partir de la mission initiale.

\subsection{Troisième condition : deux missions}

Dans les échanges de la dernière condition, on trouve deux directives explicites, pour chacun des participants. La première directive est la même que celle qui a été mise en cuvre dans la deuxième condition. La deuxième directive demande au participant de renseigner son interlocuteur sur les effets néfastes de la double cohorte. Dans ce cas, le principe de l'intention voudrait que les échanges soient difficiles, sinon impossibles. Si ce n'est pas le cas, chaque participant a pu écouter l'autre, interpréter l'information, puis réagir. Ce qu'on observe, c'est encore une fluidité des échanges. On trouve des énoncés et des réponses liés directement à un propos émis par un autre interlocuteur. Ce fait est indicatif de l'adaptabilité de l'échange. Le participant a pu modifier son discours en réponse au discours de l'autre. On observe, en moyenne, 51 énoncés qui sont reliés aux missions initiales. Cela représente environ $27 \%$ des énoncés d'un échange typique dans cette condition. En grande majorité, les propos dans cette condition ne peuvent pas être reliés à l'intention précommunicationnelle. Mais les énoncés ne sont pas sans référents. L'échange avance avec l'aide des ouvertures qui se présentent. On ne remarque pas de difficultés de la part des interlocuteurs à se faire comprendre. Ils sont en mesure de dialoguer et de répondre à des questions qui ne font pas avancer leur mission initiale. Cette flexibilité ne nuit pas à l'existence, à l'activation d'une intention déterminante et rigide. Plutôt, les interlocuteurs sont engagés et leurs discours sont expressifs. Ils font partie d'un échange qui se montre à la fois dynamique et adaptable. Prenons un exemple (tiré de l'échange PJ-26C, tours de parole numéro 157 à 168) :

157. Répondant A : Ob my god, ! c'est trop stressant...

158. Répondant $\mathrm{B}$ : C'est comme deux mois de stress consécutifs... 
159. Répondant $\mathrm{A}:$ Ouin...

160. Répondant B : Comme c'est pas...

161. Répondant $\mathrm{A}:$ Pis eee..

162. Répondant B : C'est bien trop. Je trouve qu'il devrait en ajouter. Je trouve pas que, comme c'est bien plus, ça va être bien plus relaxe comme.

163. Répondant A : Uhum...

164. Répondant B : Sure, ça va être stressant en douzième année, no matter what, il faut que tu appliques puis whatever...

165. Répondant $\mathrm{A}$ : Uhuh...

166. Répondant $\mathrm{B}$ : En treizième, c'est beaucoup plus de temps, comme, nous autres, il fallait qu'on pense à ce qu'on voulait faire...

167. Répondant $A$ : Je ne comprends pas comment ça se fait qu'ils ont éliminé la treizième... À cause, c'est une année d'extra puis t'as même pas besoin...

168. Répondant B : Puis ils l'ont pas fait au Québec...

Dans ce cas, chaque participant a une mission, celle d'informer soit des bénéfices des augmentations de frais de scolarité, soit des effets néfastes de la double cohorte. Ce qu'on remarque, d'abord, c'est que les participants expriment leur désaccord à l'égard de l'élimination de la treizième année et, donc, de la création de la double cohorte. L'échange débute avec une affirmation qu'il y a quelque chose de «trop stressant». On peut conclure qu'il est fait référence à la vie scolaire au secondaire. On doit constater que le but de cette référence est de mettre en évidence un aspect négatif de la double cohorte, soit de concentrer plus d'information dans le temps et ainsi d'augmenter le niveau de stress que vivent les étudiants. « $\mathrm{B}$ » répond sous forme de répétition à « $\mathrm{A}$ » pour montrer son accord. Comment peut-on présenter ce propos comme s'il relevait d'une logique nettement rationnelle ? Doit-on penser que « B » a répondu pour s'approcher d'un but? Si oui, quel est ce but? Ne semble-t-il pas plus probable que « $\mathrm{B}$ » réaffirme le postulat de « $\mathrm{A}$ » pour montrer son accord et pour ainsi indiquer à « $\mathrm{A}$ » qu'ils ont une opinion en commun et, par conséquent, pour donner un point de départ à la relation?

On peut se poser une autre question : où se trouve la mission de " $\mathrm{A}$ ", soit celle d'informer des bienfaits des augmentations des frais scolaires? 
On doit croire que « $A$ » témoigne d'une certaine abnégation pour écouter son partenaire, pour, en fait, se faire écouter par la suite. Est-ce possible de diverger d'une intention pour - si on croit que la réponse de « $B$ » est faite rationnellement et, par conséquent, intentionnellement - faire avancer sa mission? Si ce n'est pas le cas, le propos n'est pas uniquement rationnel. Il doit alors être émorationnel.

\subsection{1. Échange intentionné ou émorationnel}

Dans ce cas, chaque participant a une mission. Mais, en examinant l'échange, on peut remarquer clairement qu'on ne peut distinguer deux intentions ou deux rationalisations différentes. Ce qu'il y a, c'est un échange où il est question de l'augmentation du stress causée par l'élimination de la treizième année au secondaire. On a, au départ, deux intentions claires et précises; cependant, on ne peut repérer la trace que d'une seule et, comme cela a été expliqué précédemment, même cette intention ne peut être reconnue comme étant pure et sans conséquences émotives sous une forme ou une autre.

Lorsqu'on continue l'analyse des tours de paroles, on remarque que « $\mathrm{B}$ » indique que, en fait, la fin du secondaire est un moment stressant de la vie et que la fin arrive en douzième ou treizième année, cela importe peu. « $\mathrm{B}$ » avait clairement montré son appui à « $\mathrm{A}$ ». Peut-on croire que « $B$ » a maintenant changé d'opinion? Si on continue l'analyse de l'échange, on remarque un retour au postulat initial, soit celui que la diminution du temps de formation au secondaire augmente le niveau de stress chez les étudiants. Cela indique que c'est en fait le niveau de stress qu'on discute et que, en réalité, les effets néfastes de la double cohortes sont moins dramatiques que ne l'est le problème de la compression du temps d'études. Alors l'intention initiale s'est modifiée au cours de l'échange. Si la mission n'oriente pas tout à fait l'échange, il doit y avoir d'autres éléments qui contribuent à l'échange. Par conséquent, on ne peut croire que l'échange est, par essence, strictement intentionné.

\subsubsection{Des intentions qui précèdent l'échange}

Dans les échanges qu'on observe dans cette condition, on a deux intentions qui, comme le veut une logique de l'intention, ne peuvent diverger. Cependant, on remarque un éloignement des intentions initiales. Les échanges sont encore fluides. Les interlocuteurs n'ont pas 
de difficultés à interagir avec leur partenaire. Ils sont en mesure d'écouter, d'interpréter et de réagir dans cette condition aussi bien que dans les deux conditions précédentes. Les échanges sont dynamiques, uniques et imprévisibles. I a manipulation de l'intention précommunicationnelle n'a pas été appliquée au détriment de l'échange. Les interlocuteurs se sont pliés aux demandes de l'échange pour rendre la communication efficace. L'intention ne s'est pas montrée être le facteur déterminant de l'échange. L'intention a agi surtout comme un point de repère au cours des échanges, ce qui faisait en sorte que, lorsque les circonstances de l'échange le permettaient, l'intention initiale pouvait revenir à la surface.

Moins de $50 \%$ des propos, dans la troisième condition, peuvent être reliés d'une façon ou d'une autre à une intention initiale. En d'autres mots, la majorité des énoncés ne peuvent être expliqués à partir de la mission initiale. D'autres facteurs ont joué un rôle pour arriver aux échanges qu'on a observés. La capacité d'un interlocuteur de tenir un propos dans cette condition dépend surtout de la dynamique de l'échange. Dans les cas où on a observé un taux plus élevé d'énoncés relatifs au thème de la mission, on trouve des interlocuteurs engagés et passionnés à l'égard du sujet de discussion. Mais, à nouveau, les interlocuteurs n'ont pu se plier tout à fait à leur mission. Ils ont participé activement à une discussion fluide, qui n'était pas définie seulement par l'intention.

On ne peut, en grande partie, attribuer les énoncés qu'à la dynamique elle-même de l'échange. La majorité des énoncés ne proviennent pas de l'intention initiale. Ils émanent de la dynamique de l'échange, d'une combinaison de la raison, de l'émotion, du social et d'une historicité spécifique. Les réponses données par les interlocuteurs, les réactions sont toutes des indications de la nature relationnelle de la communication. Même dans un échange où tous les participants ont une intention au départ, l'intention ne parvient pas à limiter et à manipuler les discours. Elle s'insère dans la dynamique, en fait partie et aide les propos à se former ou bien elle est évoquée pour laisser s'exprimer les propos qui dérivent de la relation que génèrent les agents communicants.

\section{6. Échanges intentionnés}

Que peut-on retirer de nos résultats? À première vue, on remarque une ressemblance entre les échanges. Les discours, du moins à première vue, 
semblent tous avoir les mêmes éléments. On reconnaît des énoncés reliés à l'intention initiale, des silences ou de la répétition lorsque les interlocuteurs ne sont plus certains de ce qu'ils peuvent dire, des changements dans le discours ou même des changements dans le point de vue que prend une personne. On pourrait continuer à énumérer les uns après les autres les éléments qui se ressemblent dans les échanges, mais qui ne sont pas tout à fait pareils. Mais, en cela même, il y a le fait de la complexité des phénomènes et un appel à une théorie qui ne soit pas une sur-simplification de l'analyse.

Dans ce travail, nous avons tenté de manipuler l'intention dans le discours et, par extension, le discours lui-même. Nous avons donné une mission à certains participants pour ensuite recueillir les résultats. Ce que nous avons observé, à la suite de cette manipulation, c'est un échange imprévisible, dynamique, avec toutes ses nuances. L'intention ou la mission n'a pu contraindre l'échange ni l'empêcher. La mission semble avoir fourni un point de départ aux discussions et, à certains moments, elle a été utilisée surtout comme un point de repère, mais elle n’a pas été facteur de détermination du discours.

Ainsi une position précommunicationnelle n'agit pas comme déterminante de ces échanges. Au cours des échanges, on rencontre des réactions, des réponses, des imprévus auxquels les interlocuteurs réagissent. Ces réactions ne peuvent être de nature intentionnelle. Elles ne peuvent être détachées du contexte dans lequel elles sont formées ou de l'aspect émotif de l'être humain. L'intention doit se soumettre à ce qu'on est, à ce qui advient ; l'inverse semble moins vrai. L'humain est un être relationnel. Il fait partie d'un système complexe qui dépend de facteurs qui, pris individuellement, n'arrivent pas à expliquer le fonctionnement global du système.

L'intention des personnes qui ont participé à l'étude est imposée de l'extérieur ; elle ne leur est pas inhérente. On pourrait suggérer qu'une intention intrinsèque aurait eu une incidence différente. Elle aurait peutêtre été en elle-même plus intense. Un interlocuteur qui a un sujet à cour et qui en cerne bien les enjeux serait possiblement plus en mesure d'accomplir la tâche attendue. Cependant, peut-on justifier cette passion par la rationalité ? La passion de l'interlocuteur à l'égard du sujet n'émane-t-elle pas d'une émotion? Que la dynamique fût différente et qu'elle se compose d'une multitude de facteurs, cela n'empêche pas que, parmi ces facteurs, la rationalité et l'émotivité jouent un rôle. Il ne faut 
pas non plus oublier que bon nombre de facteurs pourraient influencer le rôle de l'intention dans la communication; qu'on songe au type même de communication, au temps qu'il y a entre l'intention et sa mise œuvre ou au temps dont dispose une personne pour réaliser son intention. Quelle que soit la variable manipulée, on ne peut croire que la manipulation éliminera l'interaction entre la rationalité et l'émotivité qu'on a observée dans cette recherche.

Enfin, on ne peut expliquer tous les propos de l'échange en fonction de la mission acceptée par l'interlocuteur, puisque lorsqu'on analyse des échanges, on n'arrive pas à trouver un fil conducteur qui pourrait relier tous les énoncés au but initial. On trouve un nombre de variables qui, prises ensemble, peuvent expliquer le discours. Néanmoins, le discours demeure plutôt imprévisible. Cette imprévisibilité a pour cause l'impossibilité de faire une analyse exhaustive de toutes les variables. On ne peut prédire le discours puisque les participants, en vertu de leur vécu qui les caractérise, du contexte dans lequel prend forme l'échange et de tous les autres éléments qui composent les grandes catégories énumérées au préalable, ne peuvent être complètement cernés.

\section{Conclusions}

\subsection{Déterminant ou élément?}

La première question à laquelle nous cherchons à répondre porte sur le rôle de l'intention dans le discours. Où se trouve l'intention dans ces échanges et quel est son rôle? La transmission d'information pour s'approcher d'un but n'est pas l'élément déterminant des échanges. On note une interaction entre les participants. On rencontre de la part de chaque participant une capacité d'adaptation qui rend la communication fluide. On ne peut ignorer la présence d'une intention. Cependant, la nature interactive des échanges fait en sorte que l'information est échangée entre les participants à partir de la dynamique qu'ils développent ou qu'ils entretiennent entre eux. Leurs propos prennent forme au cours de leur échange, ce qui indique que l'intention ne peut pas être le facteur ultime ni de détermination, ni d'explication de cette communication. C'est à l'intérieur de la dynamique entre les participants qu'on trouve l'échange d'information, et alors ce qui oriente la conversation et dont peut éventuellement faire partie une intention. 
Cette dynamique ne peut donc pas s'expliquer simplement à partir d'une intention. Elle fait appel à l'historicité des participants, à l'unicité de leur relation aussi bien qu'à l'émotion. L'échange se développe dans un contexte social et on ne peut retirer cet échange du contexte dans lequel il a pris forme. On constate bien ici à quel point il est nécessaire de recourir minimalement à une modélisation relationnelle.

Même dans les échanges intentionnés, on observe une interaction dynamique entre les participants. Alors l'intention, même si elle précède l'échange, n'en est pas la cause exclusive. Une dynamique est à l'œuvre : l'échange est composé de raison et d'émotion ; tout cela se construit dans un contexte social. Les intentions qu'on observe dans les discours prennent forme dans plusieurs contextes. Faire l'analyse d'un échange pour déterminer, en partie, d'où provient l'intention et comment elle prend forme peut se faire a posteriori. Mais l'impossibilité de prédire comment l'intention va se manifester montre bien que cette intention est moins déterminante que ne le veut la théorie qui la place en son centre. La dynamique de l'échange est bien plus déterminante du discours que l'intention elle-même. Encore, on se rend compte de la pertinence d'un modèle relationnel.

De plus, on a le cas où deux participants ont chacun, avant même que l'échange n'ait lieu, une intention. Or, on ne remarque pas de difficultés dans les échanges. Il y a fluidité. Les deux interlocuteurs se comprennent et communiquent de façon efficace. Ils écoutent, interprètent et répondent non seulement à partir de leur intention, mais aussi à partir des demandes environnementales aussi bien que des réponses émotives. Même dans le cas où les deux participants ont une intention, il y a un échange dynamique. À un certain moment, on peut retracer en partie l'intention de départ. Cependant, on la trouve difficilement à son état pur. On la retrouve plutôt à l'arrière-plan d'un échange infiniment complexe.

On doit alors avoir recours à un modèle qui prend cette dynamique en considération. Un modèle d'analyse de l'action humaine qui repose uniquement sur l'intention et sur ses corollaires ne peut pas appréhender adéquatement les échanges entre interlocuteurs. Le modèle doit prendre en considération l'aspect humain - mais dans sa complexité et non d'après ce qu'on voudrait qui le caractérise - des échanges aussi bien que le contexte social dans lequel l'échange a lieu. En d'autres mots, l'humain est plus que rationnel, il est aussi émotionnel. Il est inscrit dans 


\section{PAUL JALBERT / ANALYSE DU RÔLE DE L'INTENTION}

un contexte social dont il ne peut se retirer et, par conséquent, on ne peut en extraire son discours. On doit alors se doter d'une approche dans laquelle l'émoraison est active, c'est-à-dire une approche relationnelle qui permet d'inclure les nombreuses composantes d'un échange aussi bien que de les interpréter de façon respectueuse. Un modèle rigide ne peut satisfaire à toutes les attentes. Le modèle doit pouvoir rendre compte de la capacité qu'ont les interlocuteurs de générer leur propre échange. La catégorie émoraison permet de le faire : elle permet au moins de prendre en considération les éléments de l'échange et propose des directives pour interpréter la dynamique de cet échange. C'est avec de tels moyens qu'on peut tenter de saisir comment se déroule l'échange.

\subsection{Ouvertures}

Ces résultats obtenus, que pouvons-nous faire maintenant? Où pouvonsnous aller? Comment déterminer quelles sont les critiques d'importance de prime abord ? L'une de ces critiques devrait porter sur la construction de l'environnement dans lequel a lieu l'échange. Il nous semble qu'il serait fort intéressant de faire l'analyse des échanges dans des environnements qui pourraient être qualifiés de plus naturels. Faire des analyses de conversations qui ont eu lieu à l'extérieur du laboratoire pourrait assurer nos observations : l'analyse pourrait se donner comme objet un débat politique, où l'intention de départ est bien connue, ou même un discours militant. Dans ces cas là, on aura affaire à une mission établie et claire. Qu'en dirait une analyse ? Est-ce qu'on serait mieux à même de se suffire d'une approche rationnelle ou faudrait-t-il à nouveau faire appel à l'émoraison?

Il serait intéressant de mettre en cuvre une émotion et de faire l'analyse des conséquences sur un échange qui s'ensuivrait. De cette façon, on pourrait éliminer l'exclusivité de la rationalité comme fondement du discours et assurer la place de l'émotion et, ainsi, de l'émorationalité. 


\section{Références}

Berthelot, Jean-Michel, L'intelligence du social, Paris, PUF, 1990.

Bouchard, Pierre, Contribution à la critique de la rationalité utilitaire : pour un modèle de remplacement des théories de l'action bumaine, mémoire de maitrise, Sudbury, Université Laurentienne, Sudbury, 2000.

Caillé, Alain, Don, intérêt et désintéressement. Bourdieu, Mauss, Platon et quelques autres auteurs, Paris, La Découverte, 1994.

Caplin, Andrew et John Leahy, « Psychological expected utility theory and anticipatory feeling, The Quarterly Joumal of Economics, vol. 116, $\mathrm{n}^{\circ} 1$, 2001, p. 55-79.

Chen, Chuansheng, "Close Relationship Between Asian Americans and European American College Students: Chinese, Japanese, Korean, Vietnamese, and Filipino Amercican ", Journal of Social Psychology, vol. 141, n 1, 2001, p. 85-100.

Chou, Kee-Lee et James Chi'en, "Utility theory and adolescent drug abusers in Hong Kong », Child and Adolescent Social Work Joumal, vol. 14, n 6, 1997, p. 397-412.

Cox, James, «On testing the utility hypothesis », The Economic Joumal, vol. 107, $\mathrm{n}^{\circ} 443$, 1997, p. 1054-1078.

De Rosnay, Joël, Le macroscope. Vers une version globale, Paris, Seuil, 1975.

Feltz, Bernard, La science et le vivant. Introduction à la philosophie des sciences de la vie, Bruxelles, De Boeck, 2003.

Girard, Mélanie, Relations humaines et production d'information. L'échange comme objet d'étude d'une approcbe relationnelle, mémoire de maîtrise, Sudbury, Université Laurentienne, 2004.

Girard, Mélanie, Simon Laflamme et Pascal Roggero, "L'intention est-elle si universelle que le prétendent les théories de l'action? », Nouvelles perspectives en sciences sociales. Revue internationale de systémique complexe et d'études relationnelles, vol. 1, $\mathrm{n}^{\mathrm{o}} 2,2005$, p. 115-148.

Godbout, Jacques T., L'esprit du don, Paris, La Découverte, 1992.

Hareless, David et Colin F. Camerer, "The predictive utility of generalized expected utility theories », Econometrica, vol. 62, n 6, 1994, p. 1251-1289.

Hobbes, Thomas, Leviathan, Cambridge, Cambridge University Press, [1651] 1991.

Hollis, Martin et Robert Sugden, « Rationality in action ", Mind, vol. 405, 1993, p. 1-35.

Hume, David, A treatise of buman nature, Oxford, Clarendon Press, [1740] 1978.

Karni, Edi, "Subjective expected utility theory with state-dependant preferences ", Journal of Economic Theory, vol. 60, 1993, p. 428-438.

Laflamme, Simon, Communication et émotion. Essai de microsociologie relationnelle, Paris, L'Harmattan, 1995.

Lipman, Barton, « Decision theory without logical omniscience: Toward an Axiomatic Framework for Bounded Rationality, The Review of Economic Studies, vol. 66, $\mathrm{n}^{\circ} 2$, 1999 , p. 339-361.

Muller, Edward, Henry A. Dietz et Steven E. Finkel, « Discontent and the expected utility of rebellion: The case of Peru », The American Political Science Review, vol. 85, $\mathrm{n}^{\circ} 4,1991$, p. 1261-1282. 


\section{PAUL JALBERT / ANALYSE DU RÔLE DE L'INTENTION}

Pace, Judith, "Revisiting classroom authority: Theory and ideology meet practice ", Teachers College Record, vol. 105, $\mathrm{n}^{\circ}$ 8, 2003, p. 1559-1586.

Register, Lisa M. et Tracy B. Henley, "The phenomenology of intimacy, Journal of Social and Personal Relationships, vol. 9, $\mathrm{n}^{\circ}$ 4, 1992, p. 467-481.

Roseman, Ira J., Cynthia Wiest et Tamaea S. Swartz, « Phenomenology, behaviors, and goals differentiate discrete emotions, Journal of Personality and Social Psychology, vol. $67, \mathrm{n}^{\circ} 2,1994$, p. $206-221$.

Rousselle, Jeannine, La communication cheq les couples. Une approcbe relationnelle, mémoire de maitrise, Sudbury, Université Laurentienne, 2003.

Schmidtz, David, « Rationality within Reason », The Journal of Philosophy, vol. 89, $\mathrm{n}^{\circ}$ 9, 1992, p. 445-466.

Watzlawick, Paul, Pragmatics of human communication: A study of interactional patterns, pathologies, and paradoxes, New York, W.W. Norton, 1967.

Weirich, Paul, «Expected utility and risk, The British Journal for the Philosopby of Science, vol. $37, \mathrm{n}^{\circ} 4,1986$, p. 419-442. 